\title{
An online energy-efficient routing protocol with traffic load prospects in wireless sensor networks
}

\section{Jun Liu and Xiaoyan Hong*}

\author{
Department of Computer Science, \\ University of Alabama, \\ Tuscaloosa, AL 35487, USA \\ Email: jliu@cs.ua.edu \\ Email: hxy@cs.ua.edu \\ *Corresponding author
}

\begin{abstract}
In wireless sensor networks, many routing algorithms are designed to implement energy-efficient mechanisms. Among those, some focus on maximising an important performance index called network lifetime, which is the number of messages successfully delivered in the network before a failure. In this paper, we propose a new online algorithm taking the goal of prolonging network lifetime. When making routing decisions, our algorithm, named Traffic-Aware Energy Efficient (TAEE) routing protocol, utilises prospective traffic load information for further load balance, in addition to power-related metrics used in an enhanced cost function in calculating least cost paths. An algorithm for automatic parameter adaption is also described. To better accommodate to large-scale sensor networks, we further introduce a random grouping scheme which enables hierarchical TAEE routing to run within and cross the dynamically formed groups to reduce computation and routing overhead, while maintaining global energy efficiency. Our simulation shows that compared with the leading power-aware Max-min $z P_{\min }$ protocol, the TAEE protocol generates better performance in terms of network lifetime without jeopardising network capacity.
\end{abstract}

Keywords: wireless sensor networks; lifetime maximisation; online energy-efficient routing; traffic load awareness.

Reference to this paper should be made as follows: Liu, J. and Hong, X. (2009) 'An online energy-efficient routing protocol with traffic load prospects in wireless sensor networks', Int. J. Sensor Networks, Vol. 5, No. 3, pp.185-197.

Biographical notes: Jun Liu received the $\mathrm{PhD}$ degree in Computer Science from the University of Alabama in 2007. He is currently a Senior Analyst in Manhattan Associates. His research interests include protocol design, performance evaluation, security and privacy, routing and power-aware solutions for wireless mobile networks and sensor networks.

Xiaoyan Hong is a Professor at the Department of Computer Science at the University of Alabama, USA. She completed her PhD degree from the University of California, Los Angeles in 2003. Her research interests include wireless networks, mobile computing and wireless sensor networks.

\section{Introduction}

In recent years, Wireless Sensor Networks (WSNs) have been extensively researched and increasingly deployed in a vast range of civil, industrial and military applications. The mass production lowered the unit price, and the technical innovation boosted the sensor capabilities. However, some long-existing limitation of sensor nodes which impacts communication protocol design have not changed, e.g. most sensor nodes are still battery powered with limited energy conservation, and the wireless transmission still incurs high power consumption. At the same time, out of the lab, applications often require large-scale deployment which covers large area with complicated terrain, in which the message generation and collection may follow special requirements on quantity and frequency. This further puts challenges on communications in WSNs.
Energy efficiency of WSNs has been tackled at all the layers of communication protocol stacks. Among them, energy-efficient routing is one of the most important issues. Existing research can be categorised into classes such as: (1) least cost path-based approaches (Li et al., 2001; Toh, 2001; Hong et al., 2002; Misra and Banerjee, 2002; Akkaya and Younis, 2003; Kar et al., 2003; Gao and Zhang, 2004; Park and Sahni, 2006), (2) max-flow problem-based approaches (Chang and Tassiulas, 2000; Zussman and Segall, 2003; Chang and Tassiulas, 2004; Sadagopan and Krishnamachari, 2004), (3) data fusion and network coding approach (Heinzelman et al., 2000; Luo et al., 2005a; Ciancio et al., 2006) and (4) topology and deployment control-based approaches (Shah and Rabaey, 2002; Bogdanov et al., 2004; Kannan et al., 2004; Chatterjee et al., 2005; Kawadia and Kumar, 2005), etc. In these researches, different design objectives and metrics are addressed. Some metrics being 
measured include total power consumption, maximum throughput, the degree of power level variance of sensor nodes, cost per packet and the number of data packets extracted from the sensor nodes, etc. Many schemes seek to maximise the network lifetime. Among several definitions of lifetime, one is measured by the network's length of operation in number of successfully delivered messages until any message fails to be routed because of energy depletion. With this definition, other factors possible causing failures, such as hardware failure, noise, MAC overflow, are not counted. Energy is treated as the major reason. Another definition can be the time until the network is partitioned due to power depletion of some nodes. The former definition is especially suitable when each message is considered with equal importance in the network. In this paper, we use the former definition as our design goal.

Many existing work studies the scenario where message generation and transmission is cyclical in time, and the message distribution is uniform within a geographic field. In many other applications, data generation and transmission by the sensors are triggered by the change of metrics being monitored. In the latter scenario, event distribution is not uniform in space, and traffic can also bear a certain predictable demand through the time. In this paper, we propose a scheme that exploits the information about the sensors' prospective traffic load. The uneven distribution allows detouring routes away from hot spots in order to achieve longer lifetime. Specifically, our scheme makes route decision based on not only power-related metrics as used by many related work, but also the future traffic characteristics. This factor is significant in balancing traffic when the traffic initiation pattern is not even. In reality, this scenario is typical in many applications. For example, consider a battle field sensor network for motion detection. When a tank moves in the network area, sensor nodes near the tank tend to send more collected data in order to achieve higher precision of monitoring, while other nodes farther away may only need to transmit low-frequency data or keep in sleeping mode without sending any data at all. In addition, for large-scale sensor networks, multiple data collection base stations can be a necessity in order to satisfy requirements of fault tolerance, load balancing and efficiency. Our proposed routing scheme particularly fits in these scenarios.

Our scheme is named Traffic-Aware Energy Efficient routing protocol (TAEE) accordingly. This work extends our earlier paper (Liu and Hong, 2006) with substantial new and revised content on scheme design, analysis and evaluation results. The design goal is to maximise the network lifetime with heuristic algorithms. The routing algorithm is equipped with a new cost function using both transmission power and residual energy, with an emphasis on the power residual ratio of sensor nodes indicating risks of depletion. We further add a new metric to quantify the prospective traffic load and use it to further optimise a selected path based on the aforementioned cost function. Aligning with many existing work (e.g. Li et al., 2001) that requires each sensor node to have knowledge of the network topology and all sensor nodes' power levels, the TAEE scheme uses this dissemination mechanism and adds to it to carry the traffic load information. We expect such addition does not introduce noticeable burden to the routing process.

In addition, we take communication and computational overhead into consideration when sensor network grows large. We use traditional hierarchical routing architecture for the scalability. Our contribution is a dynamic random group mechanism to support a two-tier hierarchy. TAEE runs within the groups so it incurs less computation and communication overhead. Such design eliminates the boundary effect that occurs to grid or zone-based hierarchical solution.

In evaluating our protocol, we compare our protocol with the max-min $z P_{\text {min }}$ Algorithm (MMZ) (Li et al., 2001), a representative and leading scheme that belongs to the same category as TAEE. In MMZ, existing network status about global power consumption and residual energy is considered. The simulation results show great improvements in network lifetime under various conditions.

The rest of the paper is organised as follows. First, Section 2 gives the network model of this work. Section 3 describes the TAEE protocol in detail. We present the simulation results in Section 5. Section 6 gives a brief review of energy-efficient routing protocols for WSNs. Finally, Section 7 concludes the paper.

\section{System model}

The power consumption of a sensor node consists of computation and communication expenses. Generally, the power consumed for computation is much less than that spent on communication, with the difference typically of three orders of magnitude (Kang and Li, 2006). Thus, we focus primarily on communication power consumption in the TAEE algorithm. We then examine the computational power consumption together with communication power consumption for large-scale sensor networks. In order to reduce communication power consumption, sensor nodes are typically designed to have two states of operation: idle state and active state (Shnayder et al., 2004). In this work we only consider the optimisation of the power consumption of the sensor nodes in active state.

We view the sensor network as a graph with vertices and edges representing the sensor nodes and their direct wireless links respectively. Specifically, the graph can be represented by $G(V, E)$, with weighted vertices $V$ and edges $E$. The weights of $V$ indicate the power residual of the sensor nodes, and the weights of $E$ represent the amount of power required to transmit data between the connected sensors. According to the distance between two sensors, the power consumption required to successfully transmit a data message can be modelled as e $=k d^{c}+b$ (Heinzelman et al., 2000), where $d$ is the distance, $k, c$ and $b$ are hardware and environmentdependent constants.

The sensor network has a bootstrap phase, during which the geographical position and topological information (through beacons) is determined and disseminated. The position information can be acquired by devices like GPS, or by localisation algorithms (e.g. Bulusu et al., 2000; Sawides et al., 2001). The dissemination of these informations can be realised by broadcasting or integrated with direct diffusion 
(Intanagonwiwat et al., 2000). If the sensor network has some dynamics, e.g. topology change due to energy depletion at some nodes, mobile sinks, etc., the broadcasting can be periodic in a low rate, or change-triggered in order to reduce communication overhead.

In the bootstrap phase, together with the network topology information, nodes also distribute their initial residual energy information. Then, with the initial global view of the energy residue levels of all nodes in the network, the sink can use an all-pairs shortest path algorithm such as Floyd-Warshall algorithm (Cormen et al., 1990) or Johnson's algorithm (Johnson, 1977) to construct a shortest-path spanning tree rooted at the sink with the edge weights taking transmission power consumption into consideration. Later, the spanning tree will be used and updated in our protocol (details in Section 3).

Our design goal is to maximise the network lifetime with heuristics. The metric of lifetime is defined as the network operation length until the first failure of message delivery due to network partition or node energy depletion. The message delivery considers messages from all the sources. As we target on design an online power-aware routing scheme, it is assumed that the sequence of messages is not known for the purpose of performance optimisation, following the preconditions described by $\mathrm{Li}$ et al. (2001). With this assumption, off-line algorithms for lifetime optimisation cannot be used. It is desirable that online power-aware routing scheme can maximise the sensor network's lifetime. However, it has been proved that no algorithms running in polynomial time can be developed to solve the optimal problem to maximise lifetime (Park and Sahni, 2006). In this paper, we develop a heuristic scheme to achieve the design goal by fully exploiting the characteristics of network status in energy level, topology and traffic load pattern. Furthermore, when the network scales up, we seek to use hierarchical routing to accomplish the goal with controlled communication and computation overhead.

\section{Traffic-aware energy efficient routing protocol}

The Traffic-Aware Energy Efficient (TAEE) routing protocol is a least-cost based routing algorithm. The path selection consists of two steps. The first step is initial shortest path spanning tree construction with a cost function using powerrelated metrics including power consumption and residual energy ratio. We believe that the residual energy of a node is important when the network lifetime is concerned. An early death of a node (usually a hot spot in the network) could stop an on going traffic, leading to a reduced network lifetime. Thus, in calculating the cost function for the path selection, we seek to emphasise the influence from residual energy with a balance to the transmission energy. By doing so, we will reduce the traffic on nodes that have less energy. In the second step, we trim nodes from the resulting spanning tree using prospective traffic load and recalculate the least-cost path. This second step is equivalent to reserving power proactively at nodes en-route. In order to capture this information, we derive a new metric called prospective load ratio. In all, the TAEE protocol calculates a best path using the cost function and the new metric.

This section is organised to first introduce the metric relating to prospective traffic load, including dissemination of traffic and energy states of each node and calculation of the metric. We then describe our TAEE routing protocol, including the new cost function, the routing algorithm and the adaptivity of the algorithm. Discussions are made whenever needed.

\subsection{Traffic load pattern utilisation}

\subsubsection{Dissemination of prospective traffic load}

The knowledge of prospective traffic load at a source sensor includes its sink, data rate and its proximate lasting time. In order to utilise the information, the source must disseminate it to all the active nodes in the network which participate in routing. The active sensors can distribute this information together with the message that propagates energy level and topology information, as has been adopted by many previous works. The information update can be either periodical or instantaneous by triggering. In all, the transmission overhead for traffic load is integrated with other propagations. An example update message sent from a normal active sensor looks like $\left\langle I D_{i}, P_{i}, t t l\right\rangle$, and that from a source sensor $i$ looks like $<I D_{i}, P_{i}, t t l$, Sink $_{i}, T P_{i}, t>$.

In the messages, $I D_{i}$ is sensor node $i$ 's identity. $P_{i}$ is $i$ 's the residual power, and $\operatorname{Sink}_{i}$ is the ID of the intended sink chosen by node $i$. Here we assume each node knows its intended sink. $T P_{i}$ is the prospective traffic load in average number of packets per unit of time at sensor $i$, and $t$ indicates the effective time period of $T P_{i}$. The $t t l$ value determines the number of hops the update message should be forwarded. If deployed to large-scale sensor networks, the $t t l$ depends on the specific implementation of our hierarchical routing using random grouping to be discussed in the next subsection. From now on we call the sensor information update message SIU message. SIU messages are multicasted through the spanning tree with minimum total power consumption. After a node $j$ receives a $S I U$ message originated from the source sensor node $i$, it updates its residual energy and topology map of the network. If the $S I U$ includes source traffic information, the node $j$ also updates its prospective traffic load metric (as below). The $S I U$ message is propagated from the sources independently without synchronisation and experiences different delays to reach other sensors. Thus, sensors could have slight inconsistency about the traffic information in the whole network. Such possible inconsistency would not have a great impact on the overall routing heuristic, providing the session length is much larger than the maximum time required for $S I U$ dissemination. In addition, when the network size becomes large, the proposed dynamic grouping scheme can help control the propagation area and latency. 


\subsubsection{Prospective traffic load ratio}

For a source-destination traffic pair (source $i$ to the sink $S i n k_{i}$ ), the influence of its traffic on a node relates to the distances of this node to the source and to the destination. We use the term prospective load $(P L)$ to quantify the value. For example, for node $j$, the closer it is located to the source node $i$, or to the destination $\operatorname{Sink}_{i}$, the more likely node $j$ will be chosen to form the path for transmitting data, thus the larger value $P L$ should be. Figure 1 illustrates an example. The network has source node $S$ and the sink $D$. The bold lines indicate the shortest-path spanning tree. The double line represents the shortest-path between $S$ and $D$. The grey level of the shade covering the path gives a basic idea of how the distribution of $P L$ values contributed by the path looks like. The darker the colour, the larger the $P L$ value a node has.

Figure 1 PL values on shortest path spanning tree

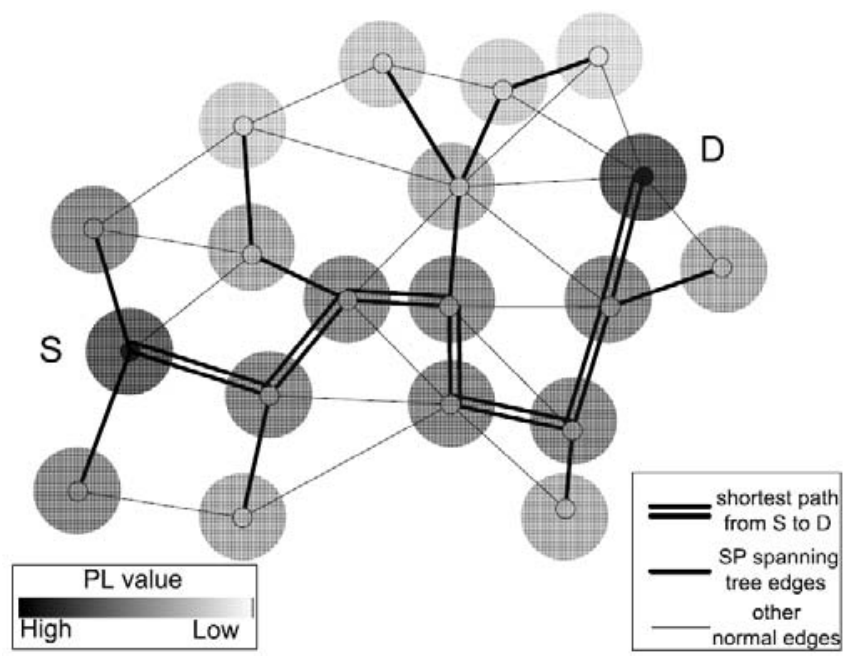

Given a path started from node $i$ with traffic load $T P_{i}$, we use formula (1) to calculate the $P L$ value at node $j$ :

$$
P L_{j}(i)=\left(f\left(d_{i, j}\right)+f\left(d_{j, \operatorname{Sink}_{i}}\right)\right) \times T P_{i}
$$

where $d_{x, y}$ represents the distance (the sum of the link costs) between node $\mathrm{x}$ and node $\mathrm{y}$ along the shortest path in the spanning tree. $f$ is a parameterised monotonically decreasing function such that $f(x) \in[0,1], f(x) \rightarrow 1$ when $x \rightarrow 0$; and $f(x) \rightarrow 0$ when $x \rightarrow x_{\max }$, where $x_{\max }$ is the maximum possible value of $x$. The essential of the $f(x)$ function is that it is monotonically decreasing and bounded in $\left[0, X_{\max }\right]$, so the variation of the output of $f(x)$ represents the impact of $x$ on the prospective traffic load.

When designing functions satisfying the above requirements, we have multiple choices which reflect the considerations for different scenarios. For example, we can have the following function:

$$
f_{1}(x) \frac{\cos \left(\left(x / x_{\max }\right) \times \pi\right)}{2}+0.5
$$

This function decreases relatively slow when $x$ drifts slightly from 0 , followed by a faster dropping speed when $x$ approaches the middle value between 0 and $x_{\max }$. When $x$ continues to increase towards $x_{\max }$, the decreasing speed again becomes low. As a second example, consider this function:

$$
f_{2}(x)=\frac{1}{x+a}-b
$$

where

$$
a=\frac{1}{2}\left(-x_{\max }+\sqrt{x^{2} \text { max }+4 x_{\max }}\right)
$$

and

$$
b=\frac{2}{-x_{\max }+\sqrt{x_{\text {max }}^{2}+4 x_{\max }}}-1 .
$$

This function decreases faster as $x$ increases when $x$ is near 0 , and slower when $x$ approaches $x_{\max }$. The slope patterns of the $f(x)$ functions interpret how the change of distance $(x)$ would impact the prospective load, and different network scenarios could have diverse matching $f(x)$ functions. For example, the $f_{1}(x)$ function is suitable for scenarios where the prospective traffic load (the PL value) on nodes is not sensitively affected by the distance (in the spanning tree) within a threshold value to the shortest path of the traffic, and when the distance exceeds the threshold, the prospective traffic load would dramatically decrease. Unlike $f_{1}(x)$, the $f_{2}(x)$ function fits into another scenario in which the prospective traffic load value is strongly bounded with distance to the shortest path (in the spanning tree). For simplicity we adopt $f_{1}(x)$ in our simulation studies.

When the network has $k$ active communication flows, node $j$ calculates the total PL value as:

$$
b=\frac{2}{-x_{\max }+\sqrt{x_{\max }^{2}+4 x_{\max }}}-1 .
$$

The PL value reflects the possible total traffic load on a node. The prospective load ratio $(P L R)$ is defined as the likelihood of this node participating in routing for a source $s$, i.e. $P L R_{j}(s)=P L_{j}(s) / P L_{j}$. PLR is utilised for node trimming and is discussed in the next subsection.

\subsection{Traffic-Aware Energy Efficient (TAEE) Routing Algorithm}

The TAEE algorithm has two main operations. The first is to construct a minimum shortest path spanning tree given an emphasis on the residual energy, and the second operation is to trim nodes from the tree according to $P L R$ value, and to recompute the path. We describe the routing algorithm as below.

We use existing algorithm like Floyd-Warshall to compute an all-pair shortest path spanning tree. The edge cost for calculating the shortest path takes the residual energy 
level and energy consumption of transmitting along the link into account. For a pair of mutual-reachable sensor node $i$ and $j$, the new weight of the edge $i \rightarrow j$ is calculated as:

$$
w_{i, j}=\frac{e_{i, j}}{\left(P_{i}-e_{i, j}\right) / P_{i_{-} \text {init }}+k}
$$

where $e_{i, j}$ is the energy required to transmit over a distance between $i$ and $j, P_{i}$ is the current power level of node $i, P_{i \text { init }}$ is the initial power level of the sensor nodes and $k$ is a non-negative parameter. We include $P_{i \text { init }}$ in the formula, considering nodes in the network could have different initial power level. The formula (2) emphasises that transmitting a message by a low residual energy node is less preferred over transmitting by a node with higher residual energy, in addition to the reduction in total path power consumption. For example, given two independent direct links that transmit a message consuming the same energy, the link with its sender having higher residual power will have a lower weight and more likely be chosen. Or say, when constructing the shortest path spanning tree, a less consumed node will be chosen. The parameter $k \geq 0$ is used to adjust the level of this emphasis. A larger $k$ results in weaker impact of the potential residual power ratio. In implementation, we choose $k=0$ for simplicity, and bound the residual power ratio to be no less than $1 \%$ of its initial power.

The routing path selection will then be optimised for load balance by using the $P L R$ value. Specifically, we use $P L R$ to trim nodes from the aforementioned spanning tree and recompute the path. For data generated by sensor $s$ and sent to the corresponding sink node $\operatorname{Sink}_{s}$, the TAEE algorithm is described in Algorithm 1 , in which $t h \in[0,1]$ is an adjustable global threshold value (an adaption algorithm will be proposed in the next subsection).

\section{Algorithm 1 TAEE Algorithm}

1 Construct the all-pairs shortest path spanning tree using Floyd-Warshall algorithm if updated SIU message is received since last transmission.

2 Find the shortest path $s p$ between $s$ and $\operatorname{Sink}_{s}$ inthe spanning tree.

3 For each node $j$ except $s$ and $\operatorname{Sink}_{s}$, and for each active transmitting source $i$, calculate $P L_{j}(i)$, total to $P L_{j}$ and calculate $P L R_{j}(s)$. If $P L R_{j}(s)<t h$, remove node $j$ temporarily from the graph only for computation in Step 4.

4 Run Dijkstra algorithm to find the shortest path from $s$ to $\operatorname{Sink}_{s}$

5 If path is found, the resulting path is the solution. Otherwise take the path generated in Step 2 as thesolution.

In Step 3, if a link does not have a large enough $P L R_{j}(s)$ corresponding to its source-sink pair $<s, \operatorname{Sink}_{s}>$ at node $j$ (i.e. $P L R_{j}(s)<t h$ ), then it is considered that other source-sink pairs are having heavier prospective load on that node. Therefore, the node $j$ will not be chosen to consist the routing path for $<s$,Sink $k_{s}>$. This says that the best shortest path linking a heavy loaded source-destination pair is protected from being assigned to forward data for a lightly loaded flow.
As a result of the algorithm, the lightly loaded flow will take a longer path than the best path it could have (when no prospective load influence is considered). Thus, our protocol prevents the sensor node that prospectively carries heavy traffic load from being added into a new path with lighter traffic load, i.e. it effectively reserves energy on that node, and it also distributes traffic to more disjoint paths.

Figure 2 gives an example illustrating the execution of the algorithm. Figure 2(a) shows that two traffic flows with source and sink pairs $\left\langle S_{1}, D_{1}>\right.$ and $\left\langle S_{1}, D_{2}>\right.$ reach a steady state. The pair $\left\langle S_{1}, D_{1}\right\rangle$ has light traffic load and $<S_{2}, D_{2}>$ has medium traffic load respectively. Figure 2(b) shows the path change of flow $<S_{2}, D_{2}>$ when $<S_{1}, D_{1}>$ pair increases traffic load from light to heavy. After the load increases, when running TAEE algorithm, it is no longer possible to include the nodes en-route of the flow $\left\langle S_{1}, D_{1}\right\rangle$ in the path for flow $<S_{2}, D_{2}>$. That is because that the $P L R_{j}$ $\left(S_{1}\right)$ ( $j$ is one of the intermediate nodes for previous $<S_{2}$, $D_{2}>$ path) resulted from the $<S_{1}, D_{1}>$ flow becomes large compared with the flow $<S_{2}, D_{2}>$ 's $P L R_{j}\left(S_{2}\right)$. Those nodes will be trimmed when calculating new paths for the flow $<S_{2}, D_{2}>$. In this case, temporarily cutting these nodes in Step 3 results in a new path from $S_{2}$ to $D_{2}$ as shown in Figure 2(b) when re-calculating a least cost path in Step 4.

Figure 2 An example of TAEE routing (see online version for colours)

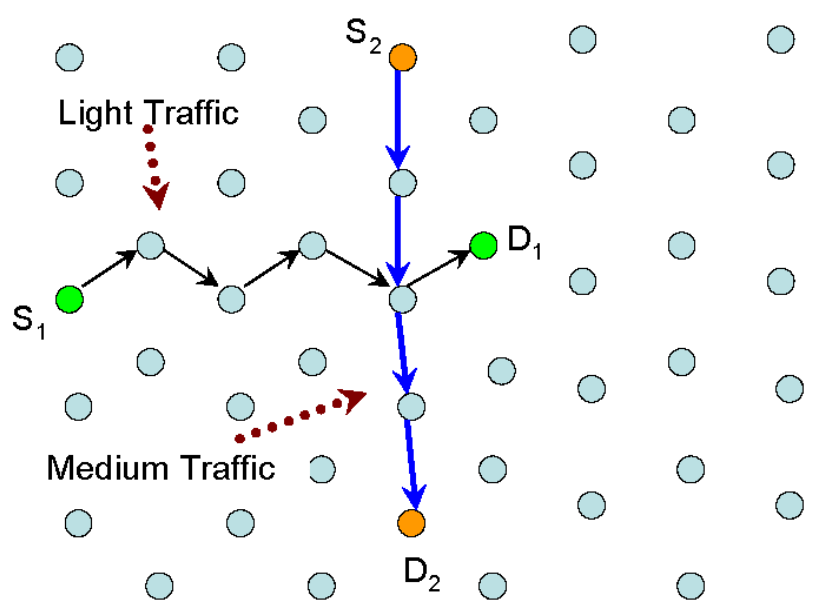

(a) Balanced Traffic Paths

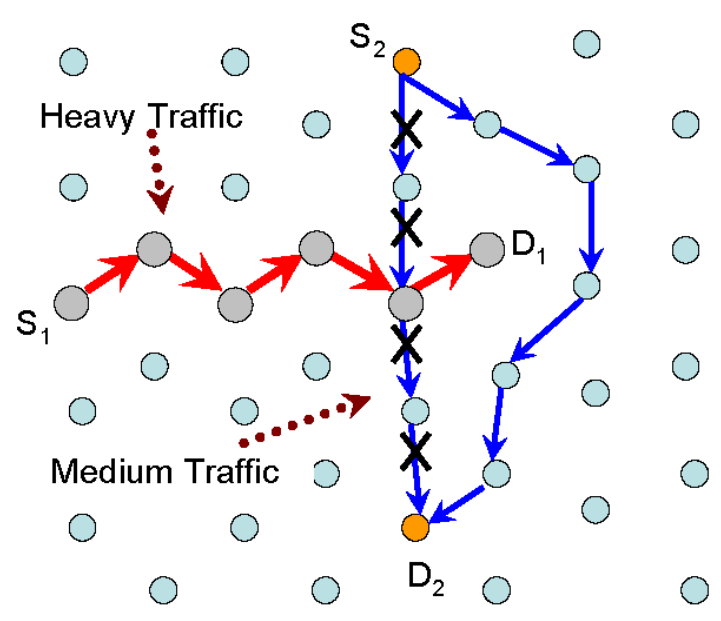

(b) Path Change Due to Traffic Load Update 
Note that when determining the nodes to remove in Step 3, we only consider the prospective traffic load ratio instead of the absolute value. To validate this measure, consider the following scenario: during the initial stage of the network lifetime, when a node has two flows potentially passing through it, even if the two flows are both light-traffic, when running the TAEE algorithm the node could be removed from the path calculation of the flow with lighter traffic due to its $P L R$ is lower than the threshold (in Step 3), while the flow with heavier traffic (even though it is still light) includes the node in its forwarding path. We observe this is reasonable measure because reserving the node for the flow with heavier traffic can prolong network lifetime. Particularly, this is true when the two flows last for long time and the node's energy could be exhausted earlier by forwarding both of them.

Another consideration is how the algorithm performs when a sink has many sources forwarding data to it. Apparently the lifetime is associated with the power level of the neighbouring nodes surrounding the sink. By TAEE the flow with heavier traffic load always takes advantage from selecting the last-hop node which results in shorter forwarding path, which means it is less likely that excessive nodes in the neighbourhood of the sink will be chosen to form paths. On the other hand, the lighter-traffic flows may need to yield to heavier-traffic flows and detour before reaching the sink. We observe this is the best routing decision available, as otherwise if the flow with heavier traffic had to detour, it would deplete the energy of the sink's neighbouring nodes faster, thus shorten the overall lifetime.

\subsection{Adaptive parameter in TAEE}

The th value is an important factor which impacts the performance of the TAEE algorithm. If $t h=1$, all nodes in the network except the source and the sink are removed in Step 3, thus no multi-hop routing is possible except the direct link if it exists. If $t h=0$, no nodes in the network is removed; therefore, the algorithm reduces to only using the cost function in formula (2) without any prospective traffic load information. In other words, if th is too small, the algorithm will fail to cut enough nodes which are prospectively heavily loaded by other source/sink pairs, and the energy reservation will become less effective.

An appropriate th value has to be adaptive with the dynamic traffic pattern. Here we use a binary-search approach to obtain the best value, an approach inspired by work of $\mathrm{Li}$ et al. (2001). TAEE with threshold adaption is shown in Algorithm 2, where $P_{n \text { init }}$ is the initial power level of nodes and $\Delta P_{t}(n)$ is the power consumed in time $t$ by node $n$. In Step 3 and Step 5, the ratio $P_{n_{-} i n i t} / \Delta P t(n)$ gives an estimate about how many time slots of $t$ a full-power node would live if the power consumption pattern follows $\Delta P_{t}(n)$ per $t$ time units. Thus, the ratio gives an intuition of the node $n$ 's lifetime when the current threshold value $t h$ is used. When the lifetime is decreasing, the threshold th needs to be changed so to remain at an optimal value.

\section{Algorithm 2 TAEE with Threshold Adaption}

1 Choose initial threshold $t h$, step $\delta$, and minimum Step $\delta_{\text {min }}>0$.

2 Run the TAEE protocol for a time interval $t$.

3 Compute $P_{n_{-} i n i t} / \Delta P t(n)$ for each node n, and let the minimum one be $l_{1}$.

4 Increase th by $\delta$ and continue to run TAEE protocol for $t$.

5 Compute $P_{n_{-} i n i t} / \Delta P t(n)$ for each node n, and let the minimum one be $l_{2}$.

6 If some node depletes its energy, stop.

7 If $l_{1}<l_{2}$, then $l_{1}=l_{2}$, goto 4 .

8 If $l_{1}>l_{2}$, then $\delta=\left\{\begin{array}{l}\max \left(-\delta / 2, \delta_{\text {min }}\right)(\text { if } \delta<0) \\ \min \left(-\delta / 2,-\delta_{\text {min }}\right)(\text { if } \delta>0)\end{array}, l_{1}=l_{2}\right.$,

goto 4

Generally, we assume the network has regular traffic pattern. That means, there is an optimal th value which maximises the network lifetime. However, when traffic pattern fluctuates, the minimum step value $\delta_{\text {min }}$ ensures the adaption step of the th does not shrink to too small, thus allowing quicker adaption when traffic fluctuation happens.

The initial step value $\delta$ and the interval $t$ are important factors for the algorithm and shall be chosen carefully. They affect the optimal th value and also the search time. A larger $\delta$ allows more global heuristic, with the sacrifice of convergence time, while a too small $\delta$ has the pitfall of being trapped at a local maximum. A smaller $t$ value allows faster threshold tuning, but the precision is limited by the statistics that can be counted in a shorter period of time. A larger $t$ allows better observation of the performance trend, but the tradeoffs are the prolonged convergence time in which the TAEE algorithm has to run with less preferable threshold value, and impact brought by the increasing change of global traffic distribution over time. In all, the parameters need to be chosen wisely according to an overall consideration of traffic periodicity, average traffic session time and transmission rate. Providing a stable traffic pattern, appropriate initial th and $\delta$, the convergence time of $t h$ is in the order of $t\left(1-\log _{2} \delta_{\min }\right)$.

\section{Hierarchical TAEE with dynamic grouping}

In many applications, sensor networks are deployed in large scale. A global propagation of residual energy and traffic distribution becomes less practical. Also, computation overhead of Algorithms 1 and 2 will rise to a higher level. To tackle this problem, we follow the traditional hierarchical approach. The key issue in such a scheme is how to form the hierarchy. Dynamic clustering and geographical based partitioning (Heinzelman et al., 2000; Li et al., 2001; $\mathrm{Xu}$ et al., 2001; Bandyopadhyay and Coyle, 2003; Luo et al., 2005b; Lin et al., 2006) are most commonly used approaches. 
The former runs a clustering protocol which incurs communication overhead, while the latter may generate boundary effect if partitions are formed statically, e.g. nodes near the borders tend to exhaust the energy quicker. In our work, we use existing geographic location information to form random zones dynamically (we call it groups) for a hierarchical routing (H-TAEE) which performs TAEE protocol locally among nodes inside the random groups.

The dynamic random grouping proposed here aims to tackle the aforementioned boundary problem and the calculation overhead. Our routing scheme remains a two-tier hierarchy using local groups. Any intermediate packet forwarders can determine the approximate directions towards the sink at any time. Using the source and the sink IDs (hence, their positions), we choose the next group in an on-demand fashion for local path selection.

A group is a sector centred at a local source with the angle $a$ and the hop range $r$. The randomness of the group is reflected in its radius $r$, its angle $a$ and its orientation $o$ (shown in Figure 3). Specifically, the local source node randomly choose a number $r \in[1, t t l]$ and uses it as the hop range for this group. $t t l$ is a constant indicating the maximum hop range. It also chooses a random angle $a \in\left[\mathrm{a}_{1}, \mathrm{a}_{2}\right]$. The sector's central line could orient towards the sink directly. We discuss these randomness a little later. The next random group (sector) will centre at a node close to the arc (called the exit node) so to forward data towards the sink. How to select this node is the job of TAEE that runs within the current group.

Figure 3 Dynamic random grouping

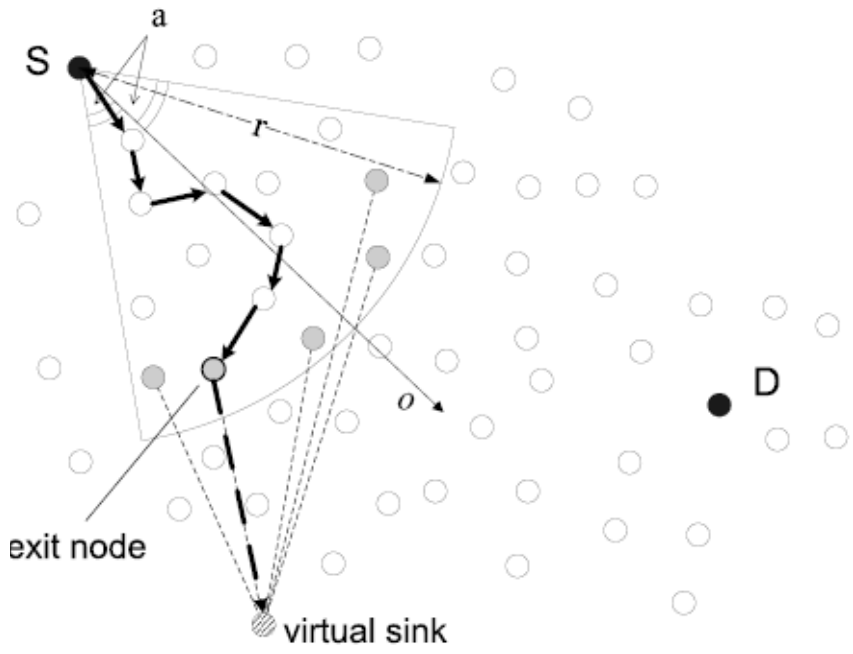

Our TAEE algorithm runs over the sensors within the sector to find a path from the local source to a node (called exit node) bordering this group on the arc with the best energy efficiency. To select the exit node, as shown in Figure 3, we connect all the candidate exit nodes (the grey nodes) to an imaginary virtual sink. The weights of the added edges are set to be 0 so the position of the virtual sink is not a concern. The TAEE will generate a heuristic energyefficient sub-path, as depicted by the bold arrowed lines in the figure. The exit node is then the node next to the virtual sink on the heuristic sub-path. Starting at this exit node, which is the local source of the next group, the next group will be formed.

We observe that the above approach of choosing the angle and the central orientation $o$ for the sector could lead to heavily using the nodes in the region (its width is determined by $a$ ) that surrounds the line linking the source and the sink (geographical shortest path). However, we are not able to use the global topological and traffic load view in calculating a fully balanced local path since our goal is to solve the scalability problem. Setting $a$ large to form a large sector for energy balance could result in large computational overhead as well. Below we describe a technique that dynamically adjusts the central orientation of the sector to alleviate the problem.

A new central orientation is calculated when the data packet is about to exit the current group. At that time, a new random group will be formed starting at the current exiting node (also the source for the next new group). The new central orientation, then, is determined based on formula (3):

$$
o_{\text {new }}=o_{\text {old }}+k_{1} \cdot a_{s \rightarrow e n}+k_{2} \cdot a_{s \rightarrow \text { sink }}
$$

where $a_{s \rightarrow e n}$ is the angle regarding to $o_{\text {old }}$ from the local source node $s$ of the current group to this exit node en, $a_{s \rightarrow \operatorname{sink}}$ is the angle regarding to $o_{\text {old }}$ from $s$ to the sink, as shown in Figure 4. $k_{1}$ and $k_{2}$ are parameters in [0,1]. The rule oscillates toward which direction the next group should be formed according to two factors. First, it exploits the trend of current group's path selection by the adjustment $k_{1}$. $a_{s \rightarrow e n}$ which predicts the orientation toward which better energy-efficiency could be achieved. A larger $k_{1}$ allows the high tier group-wise path to deviate farther from the geographical shortest path. Thus, even with a small $a$, we can achieve better energy balance. Second, it manages to converge the orientation toward the sink by adding the adjustment $k_{2} \cdot a_{s \rightarrow \operatorname{sink}}$. This adjustment is important because otherwise the route may not reach the intended sink. A possible $o_{\text {new }}$ and next group (marked by dash-dot line) starting at $e n$ are shown in Figure 4.

Figure 4 New central orientation calculation

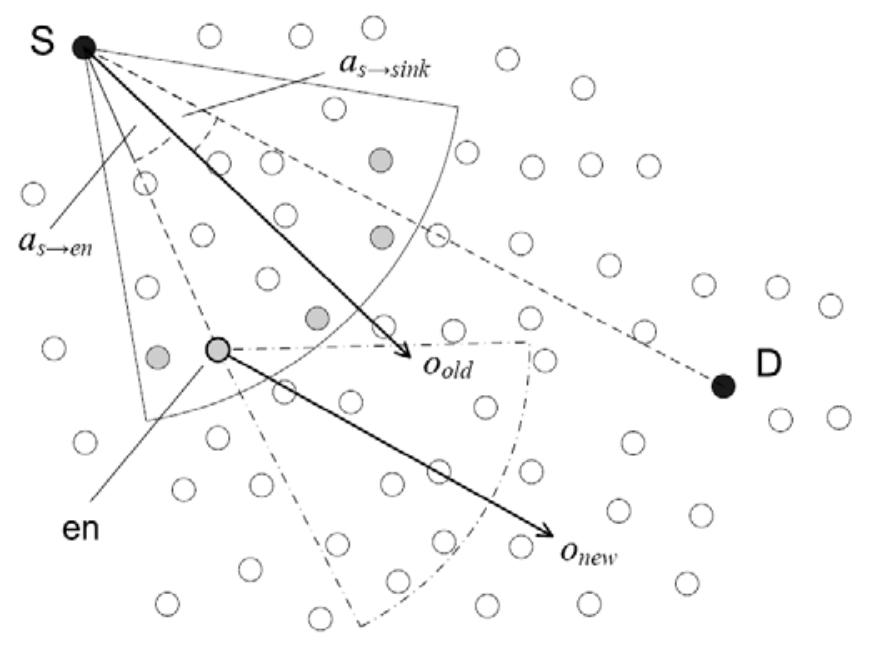


After the message is routed to the exit node in the current group, the routing process is performed repeatedly until the message reaches the destination. Because of the randomness in the group formation, the border node power depletion problem is resolved naturally. Also, because the hierarchical routing with random grouping dynamically adjusts overall orientation according to local energy-efficient path selection results, it can make effective prediction, thus accomplishing energy efficiency at the global level.

\section{Simulation}

TAEE is evaluated using a custom-built packet-level simulator and compared to the MMZ algorithm. Our primary evaluation metric is the network lifetime, which is measured as the number of messages that are transmitted from all the sources during the time period starting from the beginning until the first failure of message delivery. When appropriate we also evaluate another metric called network capacity, which is the number of messages that are transmitted from all the sources during the time period starting from the beginning until the time that no path can be established for any source-sink pairs. In addition, we study the average power expenditure per message sent from the source to the sink. The overhead of TAEE involves both communication and computation overhead. The additional communication overhead for TAEE comes from extra TP information piggybacked in normal messages propagated in the network. The resulting link overhead is limited. The computation complexity is dominated by the Floyd-Warshall algorithm as mentioned in Section 2. The Floyd-Warshall algorithm will take a complexity of $\Theta\left(N^{3}\right)$, where $N$ is the number of nodes. Our hierarchical scheme with dynamic grouping is designed to reduce the computation overhead. In addition, with the advances of the CPU processing speed, computation overhead is less concerned.

We run simulations in different scenarios where the following parameters are varied to show the impact on performance: (1) Variance of source transmission rates (we assume source sending rate is time-variable). This parameter indicates traffic load variation. Varying the degree of this variation is useful to evaluate the advantage of TAEE in networks with uneven traffic load pattern, (2) Number of nodes in the network while fixing the field size; (3) Maximum allowed transmission distance between two nodes. The value reflects the control of SNR threshold; (4) Distance between source and sink. It impacts the degree of intervention among all traffic in the network.

The network area in the simulation is square, $25 \times 25$ in size following the scenario used in early work, and the sensor nodes are deployed randomly in the area. Data sinks are distributed evenly in the sensor network, with the quantity $10 \%$ of all nodes. The data sinks change every 500 seconds. For communication we generate multiple random source-sink pairs. Five source-sink sessions are running concurrently at any time. The sessions have the same duration of 100 seconds, but with different transmission rate. The sending interval range indicates the maximum possible sending interval for any specific session which starts from 1 and selected randomly.

The equation of power consumption is defined as $e=0.001 \times d^{3}$, where $d$ is the distance between two nodes. The initial powers of the sensor nodes are randomly chosen from Johnson (1977) and Shnayder et al. (2004). These value are selected following the models from related work. Both TAEE and MMZ protocols use parameter adaption algorithms for automatically adjusting algorithm parameters. For TAEE, the threshold value is adapted according to Algorithm 2. Except being varied in the experiments or otherwise mentioned, the default configurations are set as in Table 1. When the source-sink distance is not varied, it is random because the source and sink are chosen randomly. All simulation results are averaged over simulation passes using 50 randomisation seeds.

Table 1 Default parameter configuration

\begin{tabular}{lc}
\hline Num. of Nodes & 50 \\
Trans. Range & 20 \\
E. Update Interval & $200 \mathrm{~s}$ \\
Max Sending Interval & $20 \mathrm{~s}$ \\
\hline
\end{tabular}

Figure 5 serves two purposes. We study the performance gain of TAEE over MMZ by showing the lifetime ratio. We also investigate the effectiveness of trimming nodes according to prospective traffic load (when threshold is adaptive). In comparison, we show TAEE with threshold setting to zero (no trimming will be performed). The Figure shows the impact of traffic sending rate on the above metric. Note that the maximum sending interval indicates the upper bound of the randomly generated sending interval (starting from 1) of source-destination pairs. Thus when $X$-axis moves right, the traffic becomes less even. We set the energy information update interval to be 1 for this simulation, which means both protocols always get accurate information at any time. From the figure, TAEE performs better than MMZ, no matter the threshold is adaptive or fixed at 0 . This means the cost function of TAEE balances total power consumption and maximum-minimum power residue very well, thus generates better performance. TAEE with adaptive threshold performs better than that with zero threshold, indicating the merit of exploiting traffic load information. For TAEE with adaptive threshold, it is apparent that when the sending interval range increases, which directly translates to greater level of traffic variation, the trend of network lifetime ratio is increasing near steadily. TAEE without traffic awareness $(t h=0)$ generates more irregular zigzag performance pattern as it is not directly affected by maximum sending interval. At a couple of points, it is better than TAEE with awareness due to the randomness of the traffic pattern we used. In all, Figure 5 confirms the advantage of TAEE in networks with uneven traffic patterns. 
Figure 5 Impact of traffic variation

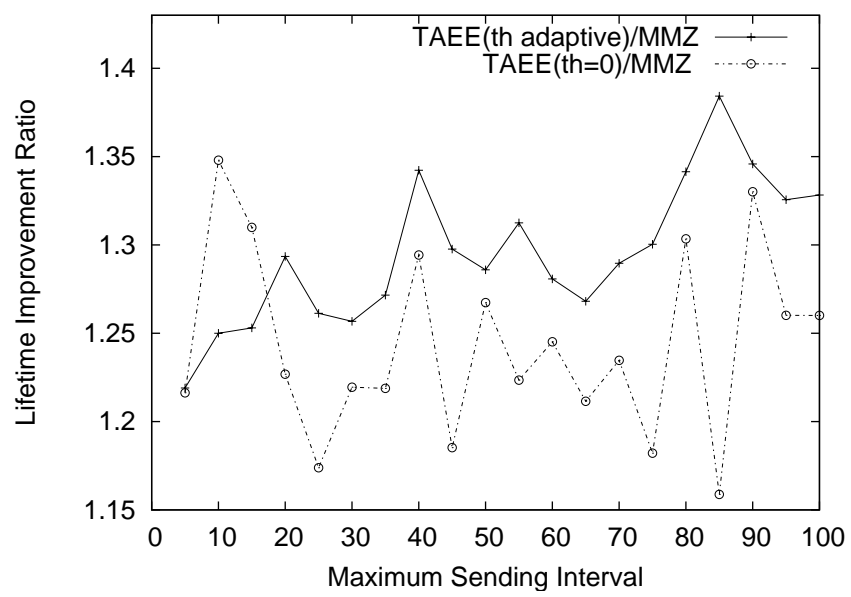

Figure 6 shows the impact of number of deployed nodes on the network lifetime. Apparently, when the number of nodes increases, the network life time is extended due to the fact that more nodes in the network create more routing path choices. Generally TAEE protocol outperforms MMZ, but when the number of nodes is small, TAEE and MMZ generates similar network lifetime. That is because in a very sparse network, the power consumption for sending a message between two nodes increases significantly along with the distance increase, causing significant reduction in number of nodes which can be removed by TAEE. For example, when selecting path for source-sink pairs with lighter traffic, the node removal (Step 3) often leaves the algorithm no choice but still to take the path calculated as shortest path, i.e. the benefit of taking traffic load vanishes. On the other hand, such a case also leaves TAEE to be more sensitive to threshold $t h$, which is more effectively adapted using the threshold adaption algorithm. However, MMZ tends to let all traffic share the same routing priority no matter the traffic session is light or not.

Figure 6 Impact of number of nodes on lifetime

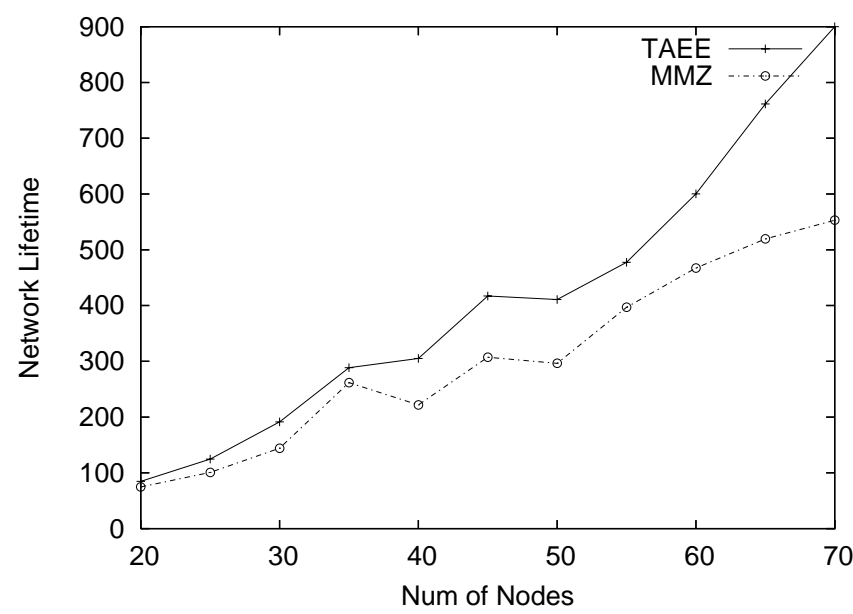

Figure 7 shows the network capacity curves for TAEE and MMZ when the number of nodes varies. The two curves are very close to each other, indicating that while TAEE has slightly higher network capacity most of the time, the performance of the two algorithms in network capacity is similar.
Figure 7 Impact of number of nodes on capacity

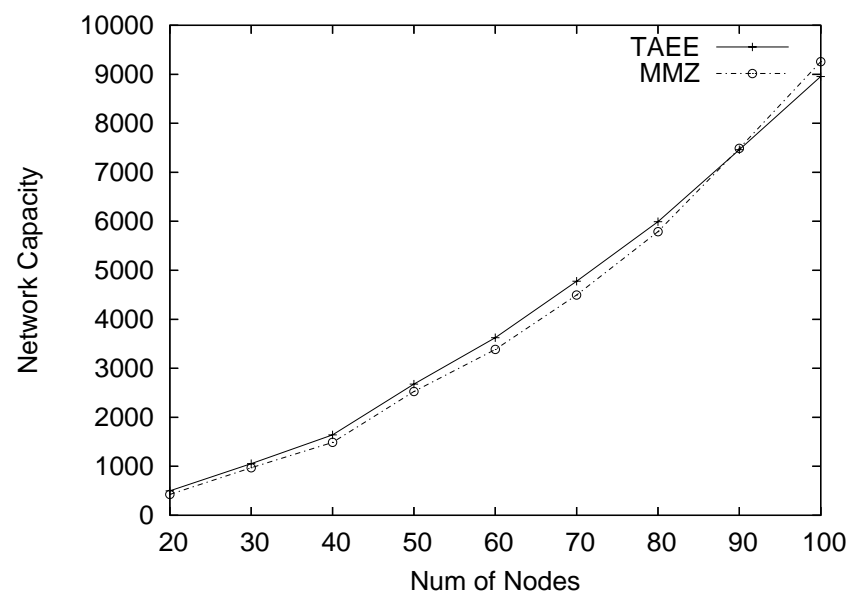

Figures 8 and 9 report average power consumption for sending a message from source to sink, from the time of traffic generation until the cut-off time for measuring network lifetime and network capacity respectively. From the two figures, most of the time TAEE has higher per-message power expenditure than MMZ, and the difference is especially obvious in the lifetime measurement scenario, and it is nearly constant when number of nodes varies. The additional power consumed by TAEE is due to the 'detouring' mechanism in the TAEE algorithm. When the number of nodes is small at 20, in the capacity measurement scenario described in Figure 9, MMZ spends more power per transmitted message than TAEE. Particularly, compared with Figure 8, when the number of nodes is 20, TAEE consumes less power while MMZ consumes more. This interesting phenomenon indicates that MMZ tends to favour paths with less overall power consumption (in addition to its power residue ratio considerations), while TAEE puts more weight on heuristic according to traffic load. Beyond the time point when the network reaches its lifetime, it is increasingly harder for TAEE to perform such heuristic by detouring, which results in the decreasing of power consumption.

Figure 8 Power expenditure per message - lifetime

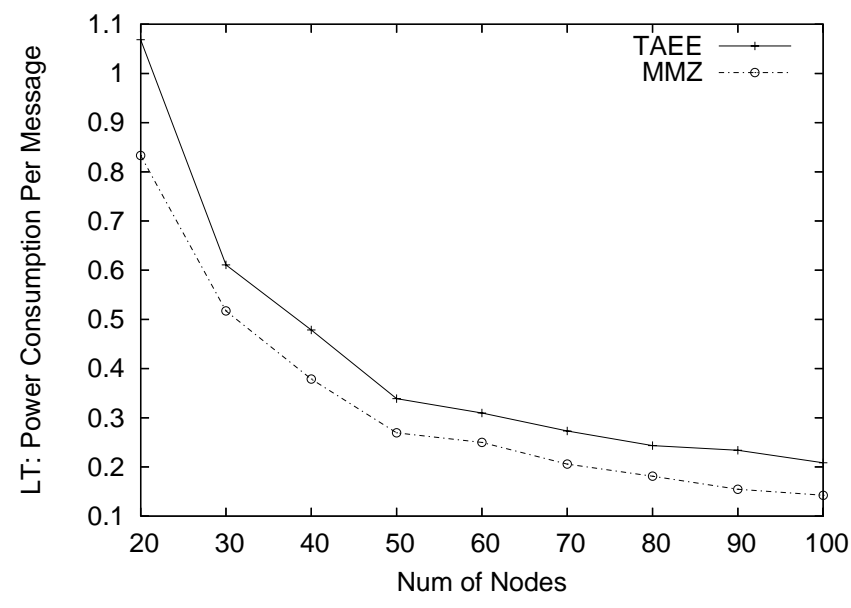

Figures 6, 7, 8 and 9 suggest that even though having a cost of a small fraction of additional power consumption, TAEE achieves longer network lifetime without jeopardising the 
network capacity. In other words, the design of detouring paths off hot spots is proved to be effective. That is to say, it achieves energy efficiency by prolonging lifetime through load balancing.

Figure 9 Power expenditure per message - capacity

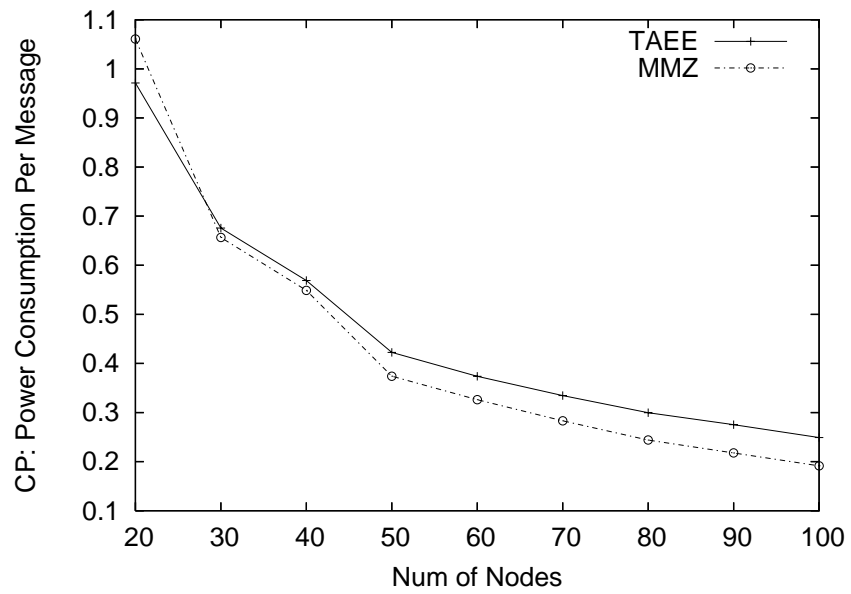

Figures 10 and 11 illustrate the impact of network transmission range. In this simulation, there are 80 nodes deployed in the network. When the transmission range is less than 3, the network is partitioned and no routes can be found. When the transmission range increases from 3 to 6 , the figures show a trend that both TAEE and MMZ increase network lifetime and network capacity. The two curves are very close because within these ranges, the network is still sparse. TAEE has the same drawback as mentioned before of not able to effectively utilising the traffic load prediction. When the range increases further, the advantage of TAEE over MMZ in network lifetime becomes obvious, and the network capacity by TAEE shows similar trends.

Figure 10 Impact of transmission range on lifetime

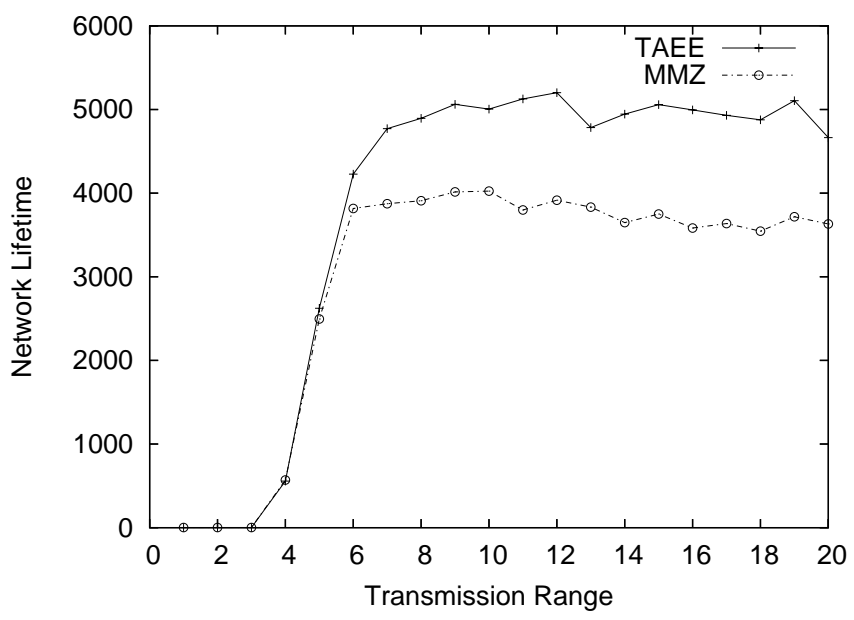

Figures 12 and 13 report the lifetime improvement ratio and capacity improvement ratio of TAEE over MMZ with varying source-sink distance range stipulated in the simulation runs. From Figure 12, the lifetime improvement ratio first increases then drops over the increase of distance. This is because when the distance is very small, the possibility of cross-traffic intervention is low. Along with the distance increased to some level, concurrent traffic flows tend to intervene with each other more intensively, which allows more benefits brought by traffic-aware TAEE protocol. When the source-sink distance continues to increase, traffic flows are more likely to cross the network area due to long path length, which increasingly restricts TAEE to find alternative detour paths. Thus the improvement ratio drops. The capacity improvement ratio described in Figure 13 follows the same trend with lower ratios.

Figure 11 Impact of transmission range on capacity

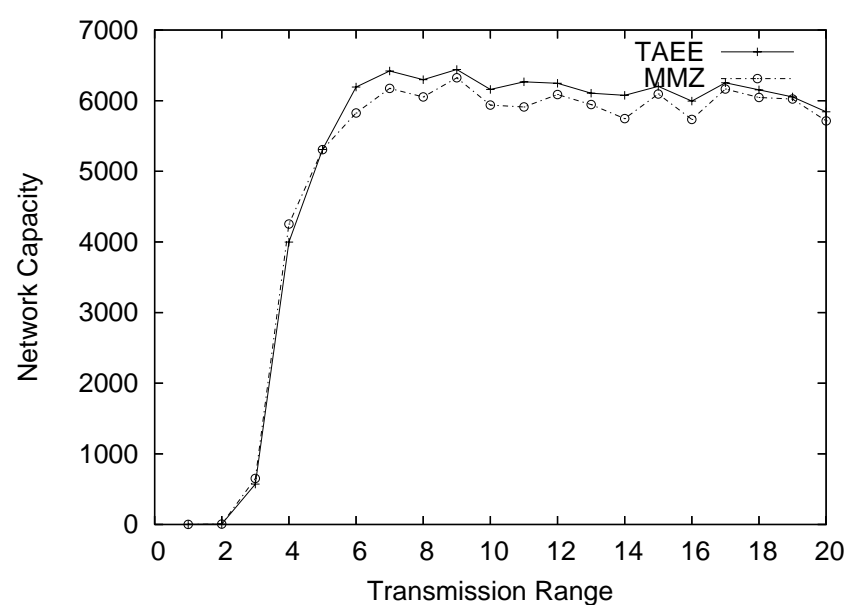

Figure 12 Impact of source-sink distance on lifetime ratio

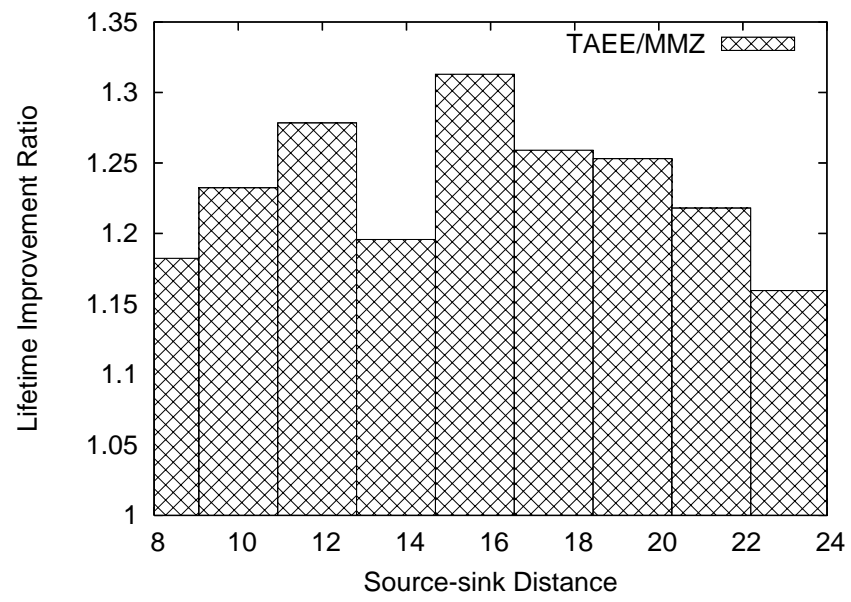

Figure 13 Impact of source-sink distance on capacity ratio

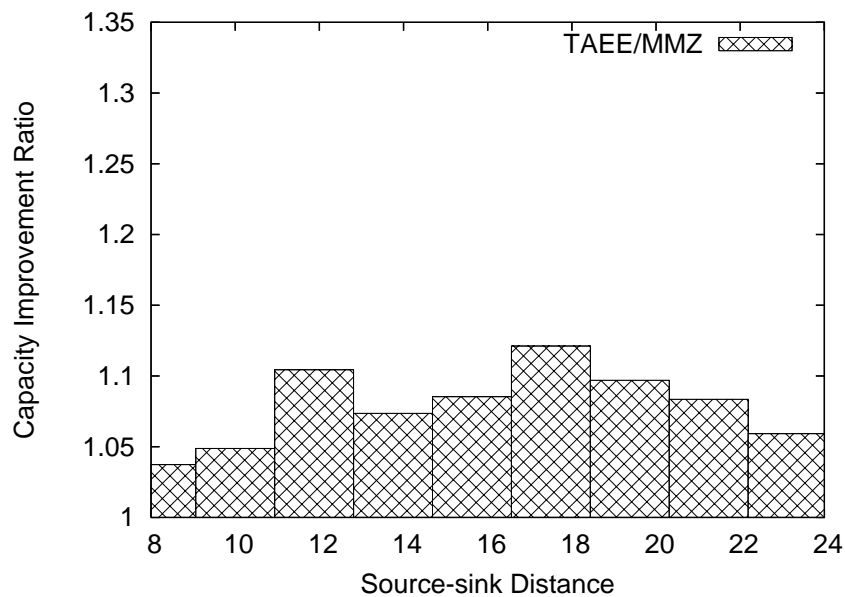


In summary, the simulation shows that our TAEE protocol generates better performance through a few mechanisms, namely, the cost function emphasising the residual energy and additional trim of potentially heavily loaded nodes. The load-balancing achieved by detouring paths can effectively prolong the network lifetime without jeopardising the capacity even with extra energy consumption per message.

\section{Related work}

Many protocols have been developed in recent years for energy-efficient communication in sensor networks. Generally, these protocols can be classified into categories including (1) Least cost path based approaches ( $\mathrm{Li}$ et al., 2001; Toh, 2001; Hong et al., 2002; Misra and Banerjee, 2002; Akkaya and Younis, 2003; Kar et al., 2003; Gao and Zhang, 2004; Park and Sahni, 2006), (2) Max-flow problem-based approaches (Chang and Tassiulas, 2000; Zussman and Segall, 2003; Chang and Tassiulas2004; Sadagopan and Krishnamachari, 2004), (3) data fusion and network coding approach (Heinzelman et al., 2000; Luo et al., 2005a; Ciancio et al., 2006) and (4) topology and deployment control based approaches (et al., 2005; Kawadia and Kumar, 2005), etc.

In least cost path-based approaches, existing works conduct online path and node selection and construct corresponding cost functions according to different criteria. A good example is the work presented by Singh et al. (1998), which proposes five metrics based on battery power consumption at nodes. By using these metrics including shortest delay, link quality, location stability, message and time overhead in route computation, the proposed protocol conducts least-cost routing to prolong the mean time of node failure. CMMBCR (Toh, 2001) performs max-lifetime heuristic routing using different metrics conditionally according to the remaining battery capacity. If there is sufficient power, shortest path algorithm is used. If not, nodes with the lowest battery capacity are avoided. Li et al. (2001) implements a heuristic algorithm Max-min $z P_{\min }$ (MMZ) to balance the power-aware metrics of minimum total power consumption and maximum minimum-power-residuefraction. MRPC (Misra and Banerjee, 2002) and EQR (Akkaya and Younis, 2003) consider not only node energy level and varied transmission power, but also packet error rates when constructing the link cost function. CMAX (Kar et al., 2003) maximises the number of messages that can be successfully delivered. It uses message admission control to bound the worst-case performance. Gao and Zhang (2004) combines greedy strategies of sending messages to the furthest reachable node-in-direction and to the node with the lightest load considering a set of special cases when nodes are located in a narrow strip. OML (Park and Sahni, 2006) proposes an online energy-aware routing scheme which initially discovers a minimum energy path, then prunes the network by discouraging the use as relays of the nodes whose current energy residue is less than the minimum energy residue along the initial minimum energy path.

Among the above class of schemes, the MMZ (Li et al., 2001) and OML (Park and Sahni, 2006) are the two algorithms with similar approaches in achieving the same design goal. MMZ is an integration of two approaches: it takes the minimum total power possible to deliver a message as $P_{\min }$, and relaxes the total power consumption to maximise the minimum residual power fraction en route. It does so by iterative trial path calculation. In each pass it removes edges (direct wireless links) which could potentially result in lower power residue ratio at a node than the lowest ratio from the previous tentative path. The procedure ends until the total power consumption upper bound is reached. By adjusting parameters, the algorithm can be reduced to a minimum total power consumption path algorithm, or max-min residual power algorithm. OML follows the similar design approach with MMZ. The difference is that it removes edges which could potentially cause the energy level of nodes to drop below the minimum energy residue along a pre-calculated shortest path. Then, it uses a cost function integrating the factors of both potential energy consumption and power residue in order to calculate the final path. No parameter adaptation is used. Both MMZ and OML perform heuristic path determination based on the observation of the network status. However, unlike the TAEE algorithm we proposed, they only consider the current network status without utilising prospective traffic load information. Without any knowledge of the data generation and transmission of the sensor nodes, the MMZ and OML algorithms are reactive protocols, that means they only respond to the current status of the network, lacking heuristic according to future network condition.

Some researchers formulate the energy-efficient problem to max-flow problems (Zussman and Segall, 2003; Bogdanov et al., 2004; Sadagopan and Krishnamachari, 2004), and (Chang and Tassiulas, 2000; Chang and Tassiulas, 2004) propose a class of Flow Augmentation (FA) algorithms and a flow redirection algorithm. In addition, protocols are designed to accomplish energy efficiency from data fusion (Heinzelman et al., 2000; Luo et al., 2005a; Ciancio et al., 2006).

Load balancing has been used in some existing work as well. LEACH (Heinzelman et al., 2000) is a clusteringbased protocol which uses randomised rotation of cluster base stations. TTDD (Luo et al., 2005b) employs source defined grids when designing a two-tier data dissemination protocol which lessens data query flooding overhead. Shah and Rabaey (2002) implements energy-aware probabilistic forwarding. Kannan et al. (2004) addresses the problem of inter-cluster routing between cluster heads by modelling the formation of paths using a game theoretic paradigm. Hong et al. (2002) uses a multi-path approach. Other approaches include energy-aware regional data dissemination ( $\mathrm{Yu}$ et al., 2001), on-demand minimum energy routing (Doshi et al., 
2002) using energy-aware link cache, usage of directional antenna to optimise control traffic and power consumption (Chatterjee et al., 2005) and power control protocols (Jung and Vaidya, 2005; Kawadia and Kumar, 2005).

\section{Conclusion}

In this paper we developed an energy efficient routing protocol for data dissemination in WSNs. The protocol takes prospective traffic load information at sensors into account when choosing a least cost path, in addition to responding to the current network energy usage. By introducing the metric of prospective load ratio and trimming nodes from a path according to the metric, our scheme is able to proactively reserves energy for sensor nodes that have continuing (to the future) data flows. We also devised a cost function that puts more weight on residual energy level. As a result, the scheme prolongs network lifetime by load balancing. Furthermore, we proposed a dynamic random grouping approach to run with a hierarchical version of TAEE. The random sectorshaped groups resolve the boundary problem pertained to the geographical zone-based grouping schemes. The two-tier TAEE reduces computation and control overhead for large scale WSNs. Our simulation results confirmed that TAEE effectively prolongs the network lifetime without jeopardising the capacity even having higher energy consumption per message when compared to a leading online energy efficient algorithm MMZ. Our future work will further explore the potential of TAEE, investigating more parameter choices of TAEE and application layer traffic scheduling along with performance comparison with more protocols.

\section{References}

Akkaya, K. and Younis, M. (2003) 'An energy-aware QoS routing protocol for wireless sensor networks', ICDCSW '03: Proceedings of the 23rd International Conference on Distributed Computing Systems, IEEE Computer Society, Washington, DC, USA, p.710.

Bandyopadhyay, S. and Coyle, E. (2003) 'An energy efficient hierarchical clustering algorithm for wireless sensor networks', Proceedings of the 22nd Annual Joint Conference of the IEEE Computer and Communications Societies (Infocom 2003).

Bogdanov, A., Maneva, E.N. and Riesenfeld, S. (2004) 'Poweraware base station positioning for sensor networks', INFOCOM.

Bulusu, N., Heideman, J. and Estrin, D. (2000, October) 'GPS-less low cost outdoor localization for very small devices', IEEE Personal Communications, Vol. 7, No. 5, pp.28-34.

Chang, J-H. and Tassiulas, L. (2000) 'Energy conserving routing in wireless ad-hoc networks', INFOCOM, pp.22-31.

Chang, J-H. and Tassiulas, L. (2004) 'Maximum lifetime routing in wireless sensor networks', IEEE/ACM Transactions on Networking, Vol. 12, No. 4, IEEE Press, Piscataway, NJ, USA, pp.609-619.
Chatterjee, S., Roy, S., Bandyopadhyay, S., Ueda, T., Iwai, H. and Obana, S. (2005) 'A power aware routing strategy for ad hoc networks with directional antenna optimizing control traffic and power consumption', IWDC, pp.275-280.

Ciancio, A., Pattern, S., Ortega, A. and Krishnamachari, B. (2006) 'Energy-efficient data representation and routing for wireless sensor networks based on a distributed wavelet compression algorithm', IPSN '06: Proceedings of the 5th International Conference on Information Processing in Sensor Networks, ACM Press, pp.309-316.

Cormen, T.H., Leiserson, C.E., and Rivest, R.L. (1990) 'The Floyd-Warshall algorithm', Section 26.2, Introduction to Algorithms, First Edition, MIT Press and McGraw-Hill, pp.558-565.

Doshi, S., Bhandare, S. and Brown, T.X. (2002) 'An on-demand minimum energy routing protocol for a wireless ad hoc network', SIGMOBILE Mobile Computing Communication Review, Vol. 6, No. 3, pp.50-66.

Gao, J. and Zhang, L. (2004) 'Load balanced short path routing in wireless networks', The 23rd Conference of the IEEE Communications Society (INFOCOM), Vol. 23, pp.1099-1108.

Heinzelman, W.R., Chandrakasan, A. and Balakrishnan, H. (2000) 'Energy-efficient communication protocol for wireless microsensor networks', HICSS '00: Proceedings of the 33rd Hawaii International Conference on System Sciences, Vol. 8, IEEE Computer Society.

Hong, X., Gerla, M., Wang, H. and Clare, L. (2002) 'Load balanced, energy-aware communications for mars sensor networks', Proceedings of IEEE Aerospace Conference, Bigsky, MT.

Intanagonwiwat, C., Govindan, R. and Estrin, D. (2000) 'Directed diffusion: a scalable and robust communication paradigm for sensor networks', Mobile Computing and Networking, pp.56-67.

Johnson, D.B. (1977, January) 'Efficient algorithms for shortest paths in sparse networks', Journal of the ACM, Vol. 24, No. 1, p.1C13.

Jung, E-S. and Vaidya, N.H. (2005) 'Power aware routing using power control in ad hoc networks', SIGMOBILE Mobile Computing Communication Review, Vol. 9, No. 3, pp.7-18.

Kang, H. and Li, X. (2006) 'Power-aware sensor selection in wireless sensor networks', Proceedings of the 5th International Conference on Information Processing in Sensor Networks (IPSN).

Kannan, R., Ray, L., Kalidindi, R. and Iyengar, S.S. (2004) 'Maxmin length-energy-constrained routing in wireless sensor networks', EWSN.

Kar, K., Kodialam, M., Lakshman, T. and Tassiulas, L. (2003) 'Routing for network capacity maximization in energyconstrained ad-hoc networks', Proceedings of IEEE INFOCOM, pp.673-681.

Kawadia, V. and Kumar, R.R. (2005) 'Principles and protocols for power control in wireless ad hoc networks', IEEE Journal on Selected Areas in Communications, Vol. 23, No. 5, pp.76-88.

Li, Q., Aslam, J. and Rus, D. (2001) 'Online power-aware routing in wireless ad-hoc networks', MobiCom '01: Proceedings of the 7th annual international conference on Mobile computing and networking, ACM Press, New York, NY, USA, pp.97-107. 
Lin, C-J., Chou, P-L. and Chou, C-F. (2006) 'HCDD: hierarchical cluster-based data dissemination in wireless sensor networks with mobile sink', IWCMC '06: Proceeding of the 2006 international conference on Communications and mobile computing, pp.1189-1194.

Liu, J. and Hong, X. (2006) 'A traffic-aware energy efficient routing protocol for wireless sensor networks', Proceedings of the 2006 IEEE International Workshop on Computer Architecture for Machine Perception and Sensing (CAMPSO6)

Luo, H., Luo, J. and Liu, Y. (2005a) 'Energy efficient routing with adaptive data fusion in sensor networks', DIALM-POMC '05: Proceedings of the 2005 joint workshop on Foundations of mobile computing.

Luo, H., Ye, F., Cheng, J., Lu, S. and Zhang, L. (2005b) 'TTDD: two-tier data dissemination in large-scale wireless sensor networks', Wireless Network, Vol. 11, Nos. 1-2, pp.161-175.

Misra, A. and Banerjee, S. (2002) 'MRPC: Maximizing network lifetime for reliable routing in wireless environments', Proceedings of IEEE Wireless Communications and Networking Conference.

Park, J. and Sahni, S. (2006, August) 'An online heuristic for maximum lifetime routing in wireless sensor networks', IEEE Transactions on Computers, Vol. 55, No. 8, pp.1048-1056.

Sadagopan, N. and Krishnamachari, B. (2004) 'Maximizing data extraction in energy-limited sensor networks', INFOCOM.

Sawides, A., Han, C.C. and Srivastava, M. (2001, July) 'Dynamic fine-grained localization in ad-hoc networks of sensors', Proceedings of Mobicom 2001, Rome, Italy.
Shah, R. and Rabaey, J. (2002) 'Energy aware routing for low energy ad hoc sensor networks', Proceedings of IEEE Wireless Communications and Networking Conference (WCNC).

Shnayder, V., Hempstead, M., Rong Chen, B., Allen, G. W. and Welsh, M. (2004) 'Simulating the power consumption of large-scale sensor network applications', SenSys '04: Proceedings of the 2nd international Conference on Embedded Networked Sensor Systems, ACM Press.

Singh, S., Woo, M. and Raghavendra, C. S. (1998) 'Power-aware routing in mobile ad hoc networks', MobiCom '98: Proceedings of the 4th annual ACM/IEEE international Conference on Mobile Computing and Networking, ACM Press, New York, NY, USA, pp.181-190.

Toh, C. (2001, June) 'Maximum battery life routing to support ubiquitous mobile computing in wireless ad hoc networks', IEEE Communications Magazine.

Xu, Y., Heidemann, J. and Estrin, D. (2001) 'Geography-informed energy conservation for ad hoc routing', Proceedings of the ACM/IEEE International Conference on Mobile Computing and Networking.

Yu, Y., Govindan, R. and Estrin, D. (2001) 'Geographical and energy aware routing: a recursive data dissemination protocol for wireless sensor networks', UCLA Computer Science Department Technical Report UCLA/CSD-TR-01-0023.

Zussman, G. and Segall, A. (2003) 'Energy efficient routing in ad hoc disaster recovery networks', Proceedings of IEEE INFOCOM. 